\title{
Effect of the lower ligand precursors on vitamin B12 production by food-grade Propionibacteria
}

\section{Chamlagain, Bhawani}

2016

Chamlagain , B , Deptula , P , Edelmann , M , Kariluoto , S , Grattepanche , F , Lacroix , C , Varmanen, P \& Piironen , V 2016, ' Effect of the lower ligand precursors on vitamin B12 production by food-grade Propionibacteria ' , LWT-Food Science and Technology , vol. 72 , pp. 117-124 . https://doi.org/10.1016/j.Iwt.2016.04.023

http://hdl.handle.net/10138/173424

https://doi.org/10.1016/j.lwt.2016.04.023

acceptedVersion

Downloaded from Helda, University of Helsinki institutional repository.

This is an electronic reprint of the original article.

This reprint may differ from the original in pagination and typographic detail.

Please cite the original version. 


\section{Accepted Manuscript}

Effect of the lower ligand precursors on vitamin B12 production by food-grade propionibacteria

Bhawani Chamlagain, Paulina Deptula, Minnamari Edelmann, Susanna Kariluoto, Franck Grattepanche, Christophe Lacroix, Pekka Varmanen, Vieno Piironen

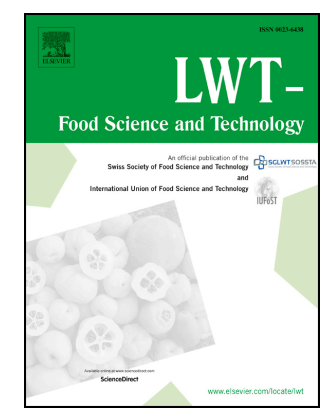

PII:

S0023-6438(16)30207-9

DOI:

10.1016/j.Iwt.2016.04.023

Reference: $\quad$ YFSTL 5411

To appear in: $\quad$ LWT - Food Science and Technology

Received Date: 1 March 2016

Revised Date: 8 April 2016

Accepted Date: 15 April 2016

Please cite this article as: Chamlagain, B., Deptula, P., Edelmann, M., Kariluoto, S., Grattepanche, F., Lacroix, C., Varmanen, P., Piironen, V., Effect of the lower ligand precursors on vitamin B12 production by food-grade propionibacteria, LWT - Food Science and Technology (2016), doi: 10.1016/ j.Iwt.2016.04.023.

This is a PDF file of an unedited manuscript that has been accepted for publication. As a service to our customers we are providing this early version of the manuscript. The manuscript will undergo copyediting, typesetting, and review of the resulting proof before it is published in its final form. Please note that during the production process errors may be discovered which could affect the content, and all legal disclaimers that apply to the journal pertain. 
1 Effect of the lower ligand precursors on vitamin B12 production by food-

2 grade propionibacteria

3

4 Bhawani Chamlagain ${ }^{1} *$, Paulina Deptula ${ }^{1}$, Minnamari Edelmann ${ }^{1}$, Susanna 5 Kariluoto $^{1}$, Franck Grattepanche ${ }^{2,3}$, Christophe Lacroix ${ }^{2}$, Pekka Varmanen ${ }^{1}$,Vieno 6 Piironen $^{1}$

$9{ }^{1}$ Department of Food and Environmental Sciences, P.O. Box 27, University of

10 Helsinki, FI-00014 University of Helsinki, Finland

$11{ }^{2}$ Laboratory of Food Biotechnology, Institute of Food, Nutrition and Health, ETH

12 Zurich, Schmelzbergstrasse 7, 8092 Zurich, Switzerland

$13{ }^{3}$ Current address: Research and Development Centre, Yoplait Ltd., Vienne,

14 France

*Corresponding author. Tel.: +358 2 94158249; fax: +358 2 94158475; E-mail:

19 bhawani.chamlagain@helsinki.fi

20 E-mail addresses of co-authors:

21 paulina.deptula@helsinki.fi; minnamari.edelmann@helsinki.fi;

22 susanna.kariluoto@helsinki.fi; franck.grattepanche@yoplait.fr;

23 christophe.lacroix@ @est.ethz.ch; pekka.varmanen@helsinki.fi;

24 vieno.piironen@helsinki.fi 
28 ABSTRACT

29 Propionibacterium freudenreichii is the only generally recognized safe (GRAS)

30 bacterium known to synthesize active vitamin B12 and offers previously untapped

31 potential for naturally fortifying foods with vitamin B12. Biosynthesis of the

32 lower ligand 5,6-dimethylbenizimidazole (DMBI) is often a key limiting factor in

33 the production of active vitamin B12 in Propionibacteria. Here, we studied the

34 effect of the natural food-grade precursors of DMBI [riboflavin (RF) and

35 nicotinamide (NAM)] on vitamin B12 production by 27 P. freudenreichii and 3

36 Propionibacterium acidipropionici strains in whey-based medium. We employed

37 sensitive and selective UHPLC and LC-MS/MS to confirm and quantify the

38 synthesized vitamin B12. In 12 P. freudenreichii strains, co-supplementation with

$39 \mathrm{RF}(40 \mu \mathrm{M})$ and NAM $(27 \mathrm{mM})$ increased the volumetric yield of vitamin B12 up

40 to 4-fold compared to the control cultures. For the majority of these strains, the

41 production level with RF and NAM exceeded the yield obtained with DMBI

42 supplementation $(100 \mu \mathrm{M})$. The significant positive correlation between RF

43 consumption and vitamin B12 production suggests that RF is proportionally

44 directed towards vitamin B12 biosynthesis. This study shows that the availability

45 of RF and NAM enhances the production of active vitamin B12 by $P$.

46 freudenreichii in a strain-dependent manner.

48 Keywords: Vitamin B12; Propionibacterium freudenreichii; fermentation;

49 riboflavin; nicotinamide 


\section{Introduction}

54 Propionibacteria are among the few bacteria and archaea that synthesize 55 vitamin B12 forms that are active for humans (hereafter called active B12)

56 (Martens, Barg, Warren, \& Jahn, 2002). The use of Propionibacterium

57 freudenreichii in foods is currently limited to the manufacture of Swiss-type 58 cheeses primarily for the characteristic eyes and typical cheese flavour (Thierry et 59 al., 2011). As the only producer of active B12 that is safe for use in food (EFSA, 60 2009), this bacterium could be utilized for the in situ B12 fortification of foods

61 and food ingredients that lack or are deficient in vitamin B12 (Hugenholtz \& 62 Smid, 2002). Genetically engineered strains of $P$. freudenreichii are used in the 63 commercial production of B12 in the pharmaceutical industry (Thierry et al., 64 2011).

65 The lower $\alpha$-ligand of B12 (5,6-dimethylbenzimidazole, DMBI) plays an 66 important role in the absorption of B12 in humans by enabling selective binding 67 of the vitamin to the intrinsic factor (a B12 transporter glycoprotein) (Alpers \& 68 Russel-Jones, 1999; Andrews, Pratt, \& Brown, 1991; Nielsen, Rasmussen, 69 Andersen, Nex $\varnothing$, \& Moestrup, 2012). For example, B12 with DMBI as the lower 70 ligand has a 500-fold higher affinity for the intrinsic factor than pseudovitamin 71 B12, which has adenine as the lower ligand (Stupperich \& Nexø, 1991). Several 72 microorganisms synthesize cobamides with other benzimidazoles, adenine or 73 phenols as the lower ligand (Watanabe, Yabuta, Tanioka, \& Bito, 2013). These 74 compounds function as cofactors for the B12-dependent enzymes in these 75 organisms (Taga \& Walker, 2008) but are not recognized by human intrinsic 76 factor (Stupperich \& Nex $\varnothing, 1991)$. 
P. freudenreichii primarily synthesizes active B12 forms with DMBI as the

78 lower ligand and adenosyl or methyl groups as the upper ligand

79 (adenosylcobalamin and methylcobalamin) but may also synthesize small

80 amounts of other cobamides, including pseudovitamin B12 (Quesada-Chanto et

81 al., 1998; Renz, 1999; Vorobjeva, 1999). Some bacteria (e.g., Lactobacillus

82 reuteri) exclusively synthesize pseudovitamin B12 even when grown with DMBI

83 supplementation (Crofts, Seth, Hazra, \& Taga, 2013; Santos et al., 2007). In a

84 recent study, $P$. freudenreichii was shown to preferentially produce active B12 in

85 a process that was guided by the biosynthesis of DMBI (Deptula et al., 2015).

86 When the availability of DMBI was restricted and therefore no active vitamin

87 could be synthesised, $P$. freudenreichii mostly accumulated incomplete cobamide

88 and only a low level of pseudovitamin B12, even when supplemented with

89 adenine (Deptula et al., 2015). The clear preference for the incorporation of

90 DMBI as the lower ligand makes $P$. freudenreichii an ideal candidate for

91 exploitation in the fermentation fortification of foods with the active B12 vitamin.

92 The complete DMBI biosynthesis pathway in aerotolerant Propionibacteria

93 was unclear for a long time. Taga, Larsen, Howard-Jones, Walsh, \& Walker (2007)

94 showed that the BluB enzyme from the soil bacterium Sinorhizobium meliloti was

95 responsible for DMBI synthesis from a reduced flavin mononucleotide in an

96 oxygenated environment. The first genome sequence of $P$. freudenreichii subsp.

97 shermanii (strain CIRM-BIA1) revealed the presence of a bluB homologue (the

98 fusion gene bluB/cobT2) (Falentin et al., 2010). Recently (Deptula et al., 2015),

99 the BluB/CobT2 fusion enzyme from $P$. freudenreichii DSM 4902 was

100 heterologously expressed, purified and characterized. The enzyme was confirmed

101 to be responsible for the synthesis of DMBI from the reduced flavin 
102 mononucleotide and its activation into the nucleotide ready for attachment as a

103 lower ligand of active B12 (Deptula et al., 2015). Flavin mononucleotide and

104 flavin-adenine dinucleotide are derived from riboflavin (RF), which together with

105 RF are collectively known as vitamin B2.

106 Natural strains of Propionibacteria differ greatly in their ability to synthesize

107 B12 (Hugenschmidt, Schwenninger, Gnehm, \& Lacroix, 2010). The B12 yield 108 from $P$. freudenreichii is dependent upon the availability of cobalt and DMBI 109 (Hugenschmidt, Schwenninger, \& Lacroix, 2011). Earlier studies with $P$. 110 freudenreichii cell homogenates showed that DMBI was synthesized from RF 111 (Lingens, Schild, Vogler, \& Renz, 1992; Renz \& Weyhenmeyer, 1972) and that 112 its biosynthesis was stimulated by nicotinamide (NAM) (Hörig \& Renz, 1980). 113 However, the effect of RF and NAM supplementation on B12 production by 114 Propionibacteria has not been studied. DMBI is added to bacterial fermentations 115 in the pharmaceutical industry to increase B12 yields (Martens et al., 2002); 116 however, this process is not possible in natural fortifications. DMBI should be 117 substituted with compounds approved for use in food (i.e., RF and NAM) or with 118 food components rich in these vitamins to enhance B12 production.

119 Therefore, the aim of the present work was to study the influence of RF and 120 NAM on B12 production by several Propionibacteria strains in whey-based 121 medium (WBM). Ultra-high performance liquid chromatography (UHPLC) and

122 liquid chromatography-tandem mass spectrometry (LC-MS/MS) were employed 123 for the accurate identification and quantification of synthesized B12 under the 124 influence of the B12 precursors. 


\subsection{Chemicals and materials}

Cyanocobalamin was obtained from Supelco (Bellefonte, USA) and ethanol was obtained from Altia (Rajamäki, Finland). Sodium hydroxide, acetic acid, dipotassium hydrogen phosphate, potassium dihydrogen phosphate, magnesium sulphate heptahydrate, manganese(II) sulphate monohydrate and granulated yeast extract were purchased from Merck (Darmstadt, Germany). Sodium cyanide, acetonitrile (HPLC grade), trifluoroacetic acid (TFA), formic acid, sodium D/Llactate syrup $(60 \% \mathrm{w} / \mathrm{w})$ and Tween 80 were obtained from Sigma-Aldrich (Steinheim, Germany). RF, NAM and cobalt(II) chloride hexahydrate were purchased from Sigma-Aldrich, and DMBI was obtained from Merck. Water (hereafter called MilliQ water) was produced by the MilliQ Plus system $(0.22 \mu \mathrm{m}$, $\geq 18.2 \mathrm{M} \Omega \mathrm{cm}$; Millipore Corporation, Bedford, MA, USA).

\subsection{Propionibacteria strains}

A collection of $27 P$. freudenreichii and three $P$. acidipropionici strains were studied. Twenty P. freudenreichii strains (256-265, 283-292) with probable dairy origins were obtained from the culture collection of Valio Ltd. (Helsinki, Finland). Two type strains (P. freudenreichii subsp. shermanii DSM 4902 and $P$. freudenreichii subsp. freudenreichii DSM 20271) with dairy origins were obtained from DSMZ (Braunschweig, Germany) and named 281 and 282, respectively. One $P$. freudenreichii strain (266) was an isolate from a cheese starter culture (Chamlagain, Edelmann, Kariluoto, Ollilainen, \& Piironen, 2015). Four $P$. freudenreichii strains (274-277) and the P. acidipropionici strains (278280) with probable cereal origins were obtained from the culture collection of Polttimo Ltd. (Lahti, Finland). 


\subsection{Growth media}

154 WBM (pH 6.4) was prepared according to Hugenschmidt et al. (2010) using demineralized whey powder (Demi 50; Valio Ltd., Helsinki, Finland) instead of

156 ultra-filtered whey permeate. The composition per $\mathrm{L}$ of the medium was $60 \mathrm{~g}$ of

157 whey extract (from whey powder), $10 \mathrm{~g}$ of yeast extract, $13 \mathrm{~g}$ of sodium D/L158 lactate syrup $(60 \% \mathrm{w} / \mathrm{w}), 0.1 \mathrm{~g}$ of Tween $80,0.2 \mathrm{~g}$ of magnesium sulphate, $0.02 \mathrm{~g}$ 159 of manganese(II) sulphate, and $100 \mathrm{~mL}$ of $1 \mathrm{M}$ potassium phosphate buffer. To 160 prepare the whey extract, an acidified whey powder suspension (85.7 g/L; pH 5.0) was autoclaved $\left(121^{\circ} \mathrm{C} ; 15 \mathrm{~min}\right)$ and paper-filtered $(10-15 \mu \mathrm{m})$. The whey extract (700 mL) was mixed with a Tween $80-\mathrm{Mg}-\mathrm{Mn}$ solution $(150 \mathrm{~mL})$ and autoclaved separately from the yeast extract, lactate and phosphate buffer solution $(150 \mathrm{~mL}$; $\mathrm{pH}$ 6.6). Immediately prior to usage, the two parts were mixed to obtain $1 \mathrm{~L}$ of medium. A filter-sterilized $(0.2 \mu \mathrm{m})$ solution of cobalt chloride was added to the medium $(5 \mathrm{mg} / \mathrm{L})$. The propionic agar medium was composed (per L) of $5 \mathrm{~g}$ of tryptone (Sigma-Aldrich), $10 \mathrm{~g}$ of yeast extract (Becton, Dickinson) and $14 \mathrm{~mL}$ of sodium lactate with the $\mathrm{pH}$ adjusted to 7.3 prior to autoclaving.

\subsection{Precursor supplementation}

171 Each strain was cultivated in triplicate in WBM with and without RF (40 $172 \mu \mathrm{M})$, the co-addition of RF $(40 \mu \mathrm{M})$ and NAM $(27 \mathrm{mM})$ or DMBI $(100 \mu \mathrm{M})$ 173 (concentrations based on Hörig \& Renz, 1980; Hugenschmidt et al., 2011). The 174 time effect of RF and NAM supplementation (on days 0,3 or 6) on B12 production was studied with strains 256 and 266. Finally, RF or RF and NAM 
176 were added on day 0 and DMBI was supplemented on day 6 per Hugenschmidt et

177 al. (2010). The stock solutions were prepared in MilliQ water and filter-sterilized.

\subsection{Culture preparation and fermentation}

Cultures of strains cryopreserved $\left(-80^{\circ} \mathrm{C}\right)$ in glycerol were propagated on propionic agar medium and incubated anaerobically (Anaerocult C; Merck, Darmstadt, Germany) for 3-4 days at $30{ }^{\circ} \mathrm{C}$. Three individual colonies of each strain were transferred into $5 \mathrm{~mL}$ of WBM and incubated for $3-4$ days at $30{ }^{\circ} \mathrm{C}$ under anaerobic conditions. The cultures were sub-cultured three times prior to inoculation. To determine B12 production by the strains, $20 \mathrm{~mL}$ of WBM was inoculated at $1 \%(\mathrm{v} / \mathrm{v})$ and incubated at $30{ }^{\circ} \mathrm{C}$ for $72 \mathrm{~h}$ under anaerobic conditions, followed by $96 \mathrm{~h}$ of aerobic incubation (with the tubes slightly opened under sterile conditions to allow air in and then closed again) under shaking conditions (150 rpm; Certomat H, Sartorious, France) (Hugenschmidt et al., 2010).

The fermented broths obtained after $168 \mathrm{~h}$ of incubation were centrifuged $(12,000 \mathrm{x} g ; 10 \mathrm{~min})$ and the supernatants were collected. The cell pellets were resuspended in $10 \mathrm{~mL}$ of PBS buffer (pH 7.3; Oxoid, Hampshire, UK) and recovered by centrifugation. The cell biomasses in the tubes were weighed and stored at $-20{ }^{\circ} \mathrm{C}$ prior to $\mathrm{B} 12$ analysis. The $\mathrm{pH}$ of the supernatants was measured; then, the supernatants were syringe-filtered $(0.2 \mu \mathrm{m}$; Pall, MI, USA) and stored at

$196-20{ }^{\circ} \mathrm{C}$ prior to analysis for residual RF, lactose and acids. The progress of 197 fermentation was monitored at $72 \mathrm{~h}$ and $168 \mathrm{~h}$ by measuring the optical density 198 (600 nm; OD $\left._{600}\right)$ with a Novespec II spectrophotometer (Amersham Pharmacia 199 Biotech, NJ, USA). The pH was recorded with a pH metre (Radiometer Analytical, 200 Lyon, France). 
The B12 content in the cell pellets was extracted in cyano form and analysed using the previously reported extraction and UHPLC methods (Chamlagain et al., 2015). Briefly, 0.1-0.2 g of cell pellet was extracted with a $\mathrm{pH} 4.5$ extraction buffer ( $8.3 \mathrm{mM}$ sodium hydroxide and $20.7 \mathrm{mM}$ acetic acid) in the presence of sodium cyanide to obtain $25 \mathrm{~mL}$ of the extract. The extract was analysed with a Waters Acquity UPLC system (Milford, MA, USA) equipped with a photodiode array detector (PDA; 210-600 nm) using an Acquity HSS T3 C18 column $(2.1 \times$ $100 \mathrm{~mm}, 1.8 \mu \mathrm{m})$. The mobile phase was a gradient flow of MilliQ water and acetonitrile both containing $0.025 \%$ TFA. The chromatogram was obtained by recording the absorbance at $361 \mathrm{~nm}$. Six cyanocobalamin standards $(0.015-0.75$ $\mathrm{ng} / \mu \mathrm{L})$ were injected (10 $\mu \mathrm{L}$ in duplicate) to create a calibration curve for each sample set. The volumetric B12 yield $(\mu \mathrm{g} / \mathrm{mL}$ WBM$)$ and cellular B12 yield $(\mu \mathrm{g} / \mathrm{g}$ wet cell mass) for each strain are reported as an average of three biological replicate fermentations.

The identity of the cobamide in the extracts was confirmed with mass spectrometry using an Esquire-LC quadrupole ion trap mass spectrometer with an electrospray ionization (ESI) interface (Bruker Daltonics, Bremen, Germany) in positive ion mode as previously described (Chamlagain et al., 2015). The mobile phase for LC-MS contained $0.1 \%$ formic acid. Briefly, ions with an $\mathrm{m} / \mathrm{z}$ range of 900-1400 were scanned, and tandem mass spectrometry (MS/MS) was performed 223 for ions with $\mathrm{m} / z 1356\left([\mathrm{M}+\mathrm{H}]^{+}\right.$of cyanocobalamin) using helium as the collision 224 gas. The instrumental settings were as follows: nebulizer (nitrogen) 50.0 psi, dry 
225 gas (nitrogen) $8.0 \mathrm{~L} / \mathrm{min}$, dry temperature $300{ }^{\circ} \mathrm{C}$, capillary $4500 \mathrm{~V}$, end plate 226 offset $-250 \mathrm{~V}$ and trap drive 84.

\subsection{Measurement of riboflavin content}

Vitamin B2 was extracted as RF from the control and fermented WBM without any supplementation and analysed using the European standard method (EN 14152:2014) after optimization for UHPLC. The supernatant $(0.5 \mathrm{~mL})$ was mixed with $15 \mathrm{~mL}$ of $0.1 \mathrm{M}$ hydrochloric acid and extracted in a boiling water

233 bath for $60 \mathrm{~min}$. After cooling, the extract was adjusted to $\mathrm{pH} 4.5$ with $2.5 \mathrm{M}$ 234 sodium acetate and incubated $\left(37^{\circ} \mathrm{C} ; 24 \mathrm{~h}\right)$ with Taka-Diastase $(50 \mathrm{mg}$; Pfaltz and 235 Bauer, CT, USA) and $\beta$-amylase ( $5 \mathrm{mg}$; Sigma-Aldrich). The analysis was performed with a Waters UPLC system equipped with a fluorescence detector using a Waters Acquity BEH C18 column $(2.1 \mathrm{~mm} \times 100 \mathrm{~mm} ; 1.7 \mu \mathrm{m}$ particles $)$.

The mobile phase contained $20 \mathrm{mM}$ ammonium acetate dissolved in $30 \%$ aqueous methanol and eluted at a constant flow of $0.2 \mathrm{~mL} / \mathrm{min}$. The excitation and emission wavelengths were set at $444 \mathrm{~nm}$ and $520 \mathrm{~nm}$, respectively. An external calibration curve was obtained by injecting six RF standards $(0.01-1.0 \mathrm{ng} / \mu \mathrm{L})$ in $10 \mu \mathrm{L}$ in duplicate for the quantitation.

\subsection{Analysis of sugar and acids}

The cell-free fermented WBM and control media were appropriately diluted with MilliQ water and filtered $(0.45 \mu \mathrm{m}$; Pall, USA). Lactose, lactic acid and the metabolites propionic acid and acetic acid in the samples were quantified with the

248 HPLC method reported by Hugenschmidt et al. (2010). The analysis was 249 performed using an HPLC system equipped with a pump (Waters 515), an 
250 autosampler, a UV detector (Waters 717) and a refractive index detector (HP

251 1047A, HP, USA) on an Aminex HPX-87H column $(7.8 \times 300 \mathrm{~mm}, 9 \mu \mathrm{m}$

252 particles; Bio-Rad, USA).

2542.9 Statistical analysis

255 The average B12 yields $(\mu \mathrm{g} / \mathrm{mL})$ and cellular B12 yields ( $\mu \mathrm{g} / \mathrm{g}$ wet cell mass)

256 for each strain following supplementation with B12 precursors were compared by

257 one way analysis of variance (ANOVA) and Tukey's post hoc test using SPSS 22

258 (IBM Corporation, NY, USA). A $p$ value $<0.05$ was considered statistically

259 significant.

260

$261 \quad 3$ Results and discussion

262

\subsection{Growth characteristics}

The final cell densities measured as the optical density at $600 \mathrm{~nm}\left(\mathrm{OD}_{600}\right)$ varied greatly between strains, ranging from $\mathrm{OD}_{600}=1.2$ to $\mathrm{OD}_{600}=17.8$ in unsupplemented WBM (Fig. 1A). A low $(<5)$ final $\mathrm{OD}_{600}$ value coincided with a high (> 6) final $\mathrm{pH}$ (Fig. 1B) that was indicative of the strain's inability to metabolize lactose in the medium. These strains relied solely on sodium lactate as a carbon source, which was also reflected in the smaller amounts of propionic and acetic acids produced by these strains (Supplemental Fig. 1A). In $P$.

270 freudenreichii, lactose utilization is strain dependent and is one of the two 271 phenotypic criteria (together with nitrate reductase activity) used to divide the species into two subspecies: subsp. freudenreichii, which is lactose negative (type

273 strain 282), and subsp. shermanii, which is lactose positive (type strain 281)

274 (Thierry et al., 2011). Five of the strains growing to higher final $\mathrm{OD}_{600}$ values 
275 (261, 262, 264, 266 and 289) produced slime during fermentation, which

276 prevented the isolation of cells from the final culture as a pellet. $P$. freudenreichii

277 strains are known to produce exopolysaccharides consisting of glucose, galactose

278 or other sugars depending on the strain (Darilmaz \& Gumustekin, 2012;

279 Nordmark, Yang, Huttunen, \& Widmalm, 2005; Thierry et al., 2011). Therefore,

280 the measured final $\mathrm{OD}_{600}$ values for these slime-producing strains most likely do 281 not reflect the final cell densities.

282 The effect of supplementation on growth was strain- and supplement283 dependent. Generally, supplementation did not restrict the growth of the strains; 284 however, co-supplementation with RF and NAM together had a stimulating effect 285 on the growth of some strains (Fig. 1A), with a few producing slime in the medium. Nevertheless, these supplements did not affect the final $\mathrm{pH}$ values of the cultures (Fig. 1B), suggesting that there was no immediate effect of carbon metabolism on acid production (Supplemental Fig. 1B).

\subsection{Confirmation of active B12 production with and without supplements}

The UHPLC-UV/Vis and LC-MS/MS analyses showed that all 27 studied strains of $P$. freudenreichii produced active $\mathrm{B} 12$ in native WBM and with RF, RF and NAM or DMBI supplementation. The cobamide extracted in its cyano form from the cell biomasses of the strains grown in WBM and DMBI-supplemented WBM eluted with the same retention time as cyanocobalamin (Fig. 2A) and had a PDA spectrum (210-600 nm) identical to cyanocobalamin (data not shown). The MS/MS spectra of the cobamide peak (Figs. 2C and 2D) were identical to the 298 fragmentation profile of cyanocobalamin (Fig. 2B). An analysis of the major 299 fragment ions $\left(\mathrm{m} / \mathrm{z} 1209,1124,997\right.$ and 912 corresponding to [M+H-DMBI] ${ }^{+}$, 
$[\mathrm{M}+\mathrm{H}-\mathrm{DMBI}-\text { sugar-phosphate-CN-Co }]^{+}$, respectively) confirmed that cobamide

302 contained DMBI as the lower ligand as reported previously (Chamlagain et al., 303 2015). Similarly, the extracted cobamide from media supplemented with RF or 304 RF and NAM was confirmed to be cyanocobalamin. In contrast, the 305 cyanocobalamin peak was detected in the cell extracts of the P. acidipropionici 306 strains only when grown with DMBI. The peak was not identified in the $P$. 307 acidipropionici strains grown with RF or with RF and NAM, confirming the 308 inability of these strains to synthesize DMBI de novo for active B12 production. DMBI is usually added during commercial B12 production; however, its use is not desirable for in situ B12 production in foods or for the production of B12 bioingredients with minimal downstream processing (Hugenschmidt et al., 2011), thereby rendering $P$. freudenreichii a more suitable candidate for these applications. However, the yield of active B12 and the response to supplementation was strain dependent (Sections 3.3-3.5).

3.3 Effect of riboflavin and nicotinamide supplementation time on B12 production

The effect of the RF and NAM supplementation time on B12 production was tested with two P. freudenreichii strains (Fig. 3). Strain 266 produced 2.4-fold more B12 when the supplementation was performed on day 0 or day 3 compared to day 6. In contrast, the B12 yield with strain 256 was not improved by RF and NAM $(p>0.05)$ but was markedly increased (by $80 \%$ ) by DMBI supplementation.

322 The results suggest that the availability of RF and NAM during the early stage of 323 fermentation improves B12 production from certain $P$. freudenreichii strains. This 324 finding is useful for the in situ B12 fortification of foods because native RF and 
325 niacin in food matrices are accessible from the beginning of the fermentation. In contrast, DMBI is preferably supplied $24 \mathrm{~h}$ before the termination of fermentation

327 in industrial B12 production (Hugenholtz, Hunik, Santos, \& Smid, 2002; Murooka,

328 Piao, Kiatpapan, \& Yamashita, 2005) because early addition of DMBI is thought

329 to reduce the growth of $P$. freudenreichii and decrease the B12 yield (Marwaha,

330 Sethi, \& Kennedy, 1983). Based on the results of this comparison, RF and NAM

331 were supplemented at the beginning of the fermentation in the latter experiment, 332 whereas DMBI was added on day 6 of the fermentation.

\subsection{B12 production in WBM without supplementation}

The B12 yield in WBM varied greatly between the studied $P$. freudenreichii strains (Figs. 4A and 4B), suggesting strain-dependent B12 production. The volumetric and cellular B12 yields ranged from 0.45 to $3.35 \mu \mathrm{g} / \mathrm{mL}$ (Fig. 4A) and 25 to $204 \mu \mathrm{g} / \mathrm{g}$ wet cell mass (Fig. 4B), respectively. For the six strains (261, 262, 264, 265, 266 and 289) that produced slime, the cellular B12 yields were not accurate and therefore were not included in Fig. 4B. Because the produced B12 accumulate intracellularly in Propionibacteria, the volumetric B12 yield $(\mu \mathrm{g} / \mathrm{mL})$ is directly affected by the amount of cell biomass obtained (data not shown) and the cellular B12 yield (Fig. 4B). Frequently, higher cellular B12 yields were obtained for strains growing to lower final $\mathrm{OD}_{600}$ values (Fig. 1A) that did not metabolize lactose while the $\mathrm{pH}$ of the medium remained $>6$ (Fig. 1B). For

346 Propionibacteria, the culture medium $\mathrm{pH}$ greatly influences growth and B12 347 biosynthesis, with pH 6-7 optimal for growth (Hsu \& Yang, 1991; Vorobjeva, 348 1999) and growth ceasing below pH 5 (Hettinga \& Reinbold, 1972). Therefore, 349 the medium is usually maintained at approximately pH 7 during industrial B12 
350 production by neutralizing the acids with alkali agents (Martens et al., 2002).

351 However, the control of $\mathrm{pH}$ to improve in situ $\mathrm{B} 12$ production in food matrices is 352 not preferred.

3543.5 Effect of riboflavin and nicotinamide vs DMBI supplementation on B12 355 production

356 RF supplementation alone did not increase B12 production by the strains $(p>$ 3570.05 ; Figs. $4 \mathrm{~A}$ and $4 \mathrm{~B})$. This result is likely due to the relatively high level $(2.5$ $358 \mu \mathrm{g} / \mathrm{mL}$ ) of RF present in the WBM. We compared the consumption of native RF 359 from the medium by the studied strains. The analyses revealed that the $P$. 360 freudenreichii strains consumed $25 \%$ to $85 \%$ of the RF present in the WBM, 361 whereas the $P$. acidipropionici strains did not use the RF from the medium. 362 Indeed, a significant positive correlation $(r=0.84)$ was observed when RF consumption was compared with B12 production (Fig. 5).

Supplementation of the WBM with both RF and NAM increased both the volumetric and cellular B12 yields in several strains ( $p<0.05$; Figs. 4A and 4B), including the $P$. freudenreichii subsp. shermanii type strain (281). Notably, this enhancement in volumetric B12 production was more effective than DMBI supplementation in seven of the strains (261-264, 266, 284 and 285) (Fig. 4A). To the best of our knowledge, the enhancing effect of RF and NAM on B12 370 biosynthesis by metabolizing $P$. freudenreichii cell has not been reported 371 previously. For four strains $(274,275,276$ and 286) and the P. freudenreichii subsp. freudenreichii type strain (282), the addition of RF and NAM significantly decreased the volumetric B12 yield $(p<0.05)$ compared to the yield in WBM. 
DMBI supplementation enhanced the volumetric and cellular B12 yields $(p<$

$3750.05)$ of seven $(256,257,281,283,288,289$ and 290) and five $(256,257,281$,

376288 and 290) P. freudenreichii strains, respectively (Figs. 4A and 4B). The

377 increase in the volumetric yield ranged from $18 \%$ for strain 257 to $280 \%$ for type

378 strain 281. The enhancement in the cellular B12 yield was most effective for

379 strain 281, with a 3.2-fold higher concentration observed in the cells with DMBI

380 supplementation compared to the control culture (Fig. 4B). Altogether, these

381 results revealed that the availability of exogenous DMBI was not limiting for B12

382 production under the conditions used for specific P. freudenreichii strains.

383 Therefore, we speculated that the $P$. freudenreichii strains that did not respond to

384 DMBI supplementation were unable to uptake DMBI from the environment. The

385 DMBI transporter in P. freudenreichii has not been identified to date.

386 By including a number of strains, the present study provides evidence in

387 support of the current understanding that P. freudenreichii synthesizes DMBI

388 from RF (Hörig \& Renz, 1977; Renz \& Weyhenmeyer, 1972; Vorobjeva, 1999).

389 The results also indicate that the efficiency of DMBI synthesis in $P$.

390 freudenreichii is strain dependent. All three $P$. acidipropionici strains studied

391 were dependent on exogenous DMBI to produce even traces of active B12 (Figs

$3924 \mathrm{~A}$ and 4B), suggesting the lack of the DMBI biosynthesis pathway in $P$.

393 acidipropionici. Indeed, genomic data (Parizzi et al., 2012) indicate that $P$.

394 acidipropionici lacks the bluB/cobT2 gene responsible for DMBI biosynthesis and

395 activation in P. freudenreichii (Deptula et al. 2015).

396 In $P$. freudenreichii, nicotinate mononucleotide (NaMN) is the preferred

397 substrate for the activation of DMBI into $\alpha$-ribazole-phosphate by the

398 BluB/CobT2 enzyme of the nucleotide loop assembly (Deptula et al., 2015; 
399 Friedmann \& Harris, 1965). NAM was rapidly converted into nicotinate in studies

400 on the DMBI-forming system using P. freudenreichii cells and cell homogenates,

401 suggesting that nicotinate was the actual stimulant of the DMBI-forming system

402 in $P$. freudenreichii (Hörig \& Renz, 1980). In line with this finding, NAM was

403 found to be completely converted into nicotinate at the end of fermentation (data

404 not shown). Thus, the synthesis or regeneration of NaMN could have been

405 enhanced by the presence of excess nicotinate. Additionally, nicotinate might

406 allosterically regulate the enzyme involved in DMBI biosynthesis (Chen, Ailion,

407 Weyand, \& Roth, 1995), which would be consistent with the greater effect of RF

408 and NAM supplementation over DMBI supplementation for the majority of the

409 strains. However, this hypothesis needs to be explored in follow-up studies.

410 In this study, the highest volumetric B12 yields (obtained with strain 288)

411 with and without DMBI supplementation were $5.3 \mu \mathrm{g} / \mathrm{mL}$ and $3.3 \mu \mathrm{g} / \mathrm{mL}$,

412 respectively (Fig. 4A). The highest yield obtained with co-supplementation with

$413 \mathrm{RF}$ and NAM was $4.2 \mu \mathrm{g} / \mathrm{mL}$ (with strain 284). All of these values clearly exceed

414 those reported in a previous study using 100 natural strains of Propionibacteria,

415 where the maximum yield in DMBI-supplemented whey permeate was $2.5 \mu \mathrm{g} / \mathrm{mL}$

416 (Hugenschmidt et al., 2010).

417

4 Conclusion

419 We used UHPLC-UV/Vis and LC-MS/MS and showed that all $27 P$.

420 freudenreichii strains studied synthesized active vitamin B12 in whey-based

421 medium without DMBI supplementation, whereas the $P$. acidipropionici strains

422 were able to produce traces of B12 only when provided exogenous DMBI. B12

423 production by the $P$. freudenreichii strains was strain dependent. The yield from 
424 several strains was markedly increased by the addition of the lower ligand

425 precursors. The volumetric B12 yield increased up to 4-fold with co-

426 supplementation with RF and NAM, and for a number of strains the yield was

427 comparable or even higher than that achieved with DMBI. A significant positive

428 correlation between RF consumption and B12 production confirmed that the

429 DMBI ligand of B12 in P. freudenreichii was synthesized from RF. The increased

430 yield obtained with RF and NAM co-supplementation clearly indicates enhanced

431 de novo synthesis of DMBI and its activation into the nucleotide. The present

432 study suggests that improved in situ production of B12 in foods is possible

433 without the need for DMBI supplementation by selecting a $P$. freudenreichii strain

434 that better responds to RF and NAM.

Acknowledgements

This study was funded by the Academy of Finland (grant no 257333) and the

Finnish Graduate School on Applied Sciences: Bioengineering, Food \& Nutrition,

Environment (ABS). We thank Miikka Olin for technical assistance during the

UHPLC and MS analyses.

\section{References}

Alpers, D. V., \& Russel-Jones, G. (1999). Intrinsic factor, haptocorrin and their receptors. In R. Banerjee (Ed.), Chemistry and Biochemistry of B12 (pp. 411-440). New York: Jhon Wiley \& Sons Inc.

Andrews, E. R., Pratt, J. M., \& Brown, K. L. (1991). Molecular recognition in the binding of vitamin B12 by the cobalamin-specific intrinsic factor. FEBS Letters, 281, 90-92.

Chamlagain, B., Edelmann, M., Kariluoto, S., Ollilainen, V., \& Piironen, V. (2015). Ultra-high performance liquid chromatographic and mass spectrometric analysis of active vitamin B12 in cells of Propionibacterium and fermented cereal matrices. Food Chemistry, 166, 630-638. doi:10.1016/j.foodchem.2014.06.068

Chen, P., Ailion, M., Weyand, N., \& Roth, J. (1995). The end of the cob operon: Evidence that the last gene (cobT) catalyzes synthesis of the lower ligand of vitamin B12, dimethylbenzimidazole. Journal of Bacteriology, 177(6), 1461-1469.

Crofts, T. S., Seth, E. C., Hazra, A. B., \& Taga, M. E. (2013). Cobamide structure depends on both lower ligand availability and CobT substrate specificity. Chemistry and Biology, 20, 12651274. doi:10.1016/j.chembiol.2013.08.006 
Darilmaz, D. O., \& Gumustekin, Y. (2012). Research on some factors influencing acid and exopolysaccharide produced by dairy Propionibacterium strains isolated from traditional homemade Turkish cheeses. Journal of Food Protection, 75(5), 918-926. doi:10.4315/0362028X.JFP-11-510

Deptula, P., Kylli, P., Chamlagain, B., Holm, L., Kostiainen, R., Piironen, V., et al. (2015). BluB/CobT2 fusion enzyme activity reveals mechanisms responsible for production of active form of vitamin B12 by Propionibacterium freudenreichii. Microbial Cell Factories, 14(1), 186. doi:10.1186/s12934-015-0363-9

EFSA. (2009). Scientific opinion on the maintenance of the list of QPS microorganisms intentionally added to food or feed (2009 update). EFSA Journal, 7(12), 1431. doi:10.2903/j.efsa.2009.1431.

EN 14152:2014 Foodstuffs - Determination of vitamin B2 by high performance liquid chromatography.

Falentin, H., Deutsch, S. M., Jan, G., Loux, V., Thierry, A., Parayre, S., et al. (2010). The complete genome of Propionibacterium freudenreichii CIRM-BIA1T, a hardy actinobacterium with food and probiotic applications. PLOS ONE, 5(7), e11748. doi:10.1371/journal.pone.0011748

Friedmann, H. C., \& Harris, D. L. (1965). The formation of $\alpha$-glycosidic 5'-nucleotides by a single displacement trans-N-glycosidase. The Journal of Biological Chemistry, 240(1), 406-12.

Hettinga, D. H., \& Reinbold, G. W. (1972). Propionic-acid bacteria- a review. 1. Growth. Journal of Milk and Food Technology, 35(5), 295-301.

Hörig, J., \& Renz, P. (1977). Biosynthesis of vitamin B12. Formation of free 5,6dimethylbenzimidazole and alpha-ribazole from riboflavin by Propionibacterium freudenreichii. FEBS Letters, 80(2), 337-339. doi:10.1016/0014-5793(77)80470-5

Hörig, J. A., \& Renz, P. (1980). Biosynthesis of vitamin B12: Some properties of the 5,6dimethylbenzimidazole-forming system of Propionibacteirum freudenreichii and Propionibacterium shermanii. European Journal of Biochemistry, 105, 587-592.

Hsu, S.-T., \& Yang, S.-T. (1991). Propionic acid fermentation of lactose by Propionibacterium acidipropionici: Effects of pH. Biotechnology and Bioengineering, 38(6), 571-578.

Hugenholtz, J., Hunik, J., Santos, H., \& Smid, E. (2002). Nutraceutical production by propionibacteria. Lait, 82, 103-112. doi:10.1051/lait:2001009

Hugenholtz, J., \& Smid, E. J. (2002). Nutraceutical production with food-grade microorganisms. Current Opinion in Biotechnology, 13, 497-507. doi:10.1016/S0958-1669(02)00367-1

Hugenschmidt, S., Schwenninger, S. M., Gnehm, N., \& Lacroix, C. (2010). Screening of a natural biodiversity of lactic and propionic acid bacteria for folate and vitamin B12 production in supplemented whey permeate. International Dairy Journal, 20(12), 852-857. doi:10.1016/j.idairyj.2010.05.005

Hugenschmidt, S., Schwenninger, S. M., \& Lacroix, C. (2011). Concurrent high production of natural folate and vitamin B12 using a co-culture process with Lactobacillus plantarum SM39 and Propionibacterium freudenreichii DF13. Process Biochemistry, 46(5), 10631070. doi:10.1016/j.procbio.2011.01.021

Lingens, B., Schild, T. A., Vogler, B., \& Renz, P. (1992). Biosynthesis of vitamin B12: Transformation of riboflavin $2 \mathrm{H}$-labeled in the 1 ' $\mathrm{R}$ position or 1 'S position into 5,6dimethylbenzimidazole. European Journal of Biochemistry, 207, 981-985. doi:10.1111/j.1432-1033.1992.tb17133.x

Martens, J. H., Barg, H., Warren, M. J., \& Jahn, D. (2002). Microbial production of vitamin B12. Applied Microbiology and Biotechnology, 58, 275-285. doi:10.1007/s00253-001-0902-7

Marwaha, S. S., Sethi, R. P., \& Kennedy, J. F. (1983). Influence of 5,6-dimethylbenzimidazole (DMB) on vitamin B12 biosynthesis by strains of Propionibacterium. Enzyme and Microbial Technology, 5(5), 361-364. 
Murooka, Y., Piao, Y., Kiatpapan, P., \& Yamashita, M. (2005). Production of tetrapyrrole compounds and vitamin B12 using genetically engineering of Propionibacterium freudenreichii. An overview. Lait, 85, 9-22. doi:10.1051/lait:2001035

Nielsen, M. J., Rasmussen, M. R., Andersen, C. B. F., Nexø, E., \& Moestrup, S. K. (2012). Vitamin B12 transport from food to the body's cells-a sophisticated, multistep pathway. Nature Reviews Gastroenterology \& Hepatology, 9(6), 345-354. doi:10.1038/nrgastro.2012.76

Nordmark, E.-L., Yang, Z., Huttunen, E., \& Widmalm, G. (2005). Structural studies of the exopolysaccharide produced by Propionibacterium freudenreichii ssp. shermanii JS. Biomacromolecules, 6(1), 521-523.

Parizzi, L. P., Grassi, M. C. B., Llerena, L. A., Carazzolle, M. F., Queiroz, V. L., Lunardi, I., et al. (2012). The genome sequence of Propionibacterium acidipropionici provides insights into its biotechnological and industrial potential. BMC Genomics, 13, 562. doi:10.1186/14712164-13-562

Quesada-Chanto, A., Schmid-Meyer, A., Schroeder, A., Fuchter, A., Carvalho-Jonas, M., Koehntopp, P., \& Jonas, R. (1998). Comparison of methods for determination of vitamin B12 in microbial material. Biotechnology Techniques, 12(1), 75-77.

Renz, P. (1999). Biosynthesis of the 5,6-dimethylbenzimidazole moiety of cobalamin and of the other bases found in natural corrinoids. In R. Banerjee (Ed.), Chemistry and Biochemistry of $B 12$ (pp. 557-575). New York: Jhon Wiley \& Sons Inc.

Renz, P., \& Weyhenmeyer, R. (1972). Biosynthesis of 5,6-dimethylbenzimidazole from riboflavin: Transformation of C-1' of riboflavin into C-2 of 5,6-dimethylbenzimidazole. FEBS Letters, 22(1), 124-126.

Santos, F., Vera, J. L., Lamosa, P., de Valdez, G. F., de Vos, W. M., Santos, H., et al. (2007). Pseudovitamin B12 is the corrinoid produced by Lactobacillus reuteri CRL1098 under anaerobic conditions. FEBS Letters, 581, 4865-4870. doi:10.1016/j.febslet.2007.09.012

Stupperich, E., \& Nexø, E. (1991). Effect of the cobalt-N coordination on the cobamide recognition by the human vitamin B12 binding proteins intrinsic factor, transcobalamin and haptocorrin. European Journal of Biochemistry, 199, 299-303.

Taga, M. E., Larsen, N. A., Howard-Jones, A. R., Walsh, C. T., \& Walker, G. C. (2007). BluB cannibalizes flavin to form the lower ligand of vitamin B12. Nature, 446, 449-453. doi:10.1038/nature05611

Taga, M. E., \& Walker, G. C. (2008). Pseudo-B12 joins the cofactor family. Journal of Bacteriology, 190(4), 1157-1159. doi:10.1128/JB.01892-07

Thierry, A., Deutsch, S. M., Falentin, H., Dalmasso, M., Cousin, F. J., \& Jan, G. (2011). New insights into physiology and metabolism of Propionibacterium freudenreichii. International Journal of Food Microbiology, 149, 19-27. doi:10.1016/j.ijfoodmicro.2011.04.026

Vorobjeva, L. I. (1999). Propionibacteria. Dordrecht, The Netherlands: Kluwer Academic Publishers.

Watanabe, F., Yabuta, Y., Tanioka, Y., \& Bito, T. (2013). Biologically active vitamin B12 compounds in foods for preventing deficiency among vegetarians and elderly subjects. Journal of Agricultural and Food Chemistry, 61, 6769-6775. doi:10.1021/jf401545z

\section{Figure captions:}

Fig. 1. Effect of riboflavin (RF), co-supplementation with RF and nicotinamide (NAM), and DMBI on the optical density (4A) and $\mathrm{pH}(\mathbf{4 B})$ of whey-based 
553 medium (WBM) fermented with 30 Propionibacterium strains. DMBI = 5,6-

554 dimethylbenzimidazole.

555 Fig. 2. A) Example UHPLC-UV chromatograms of the cyanocobalamin standard 556 and extracts of the cell biomass obtained from 168-h fermented whey-based 557 medium (WBM) and from WBM supplemented with DMBI. LC-MS/MS spectra 558 of the cyanocobalamin standard (B) and the cobamide in cell extracts eluting at 559 the retention time of cyanocobalamin $(3.27 \mathrm{~min})$ from cell biomasses grown in 560 WBM (C) and WBM with DMBI supplementation (D). DMBI = 5,6561 dimethylbenzimidazole.

562 Fig. 3. Effect of the riboflavin (RF) and nicotinamide (NAM) addition time (days 5630,3 or 6) on vitamin B12 production by P. freudenreichii strains 256 and 266 in 564 whey-based medium (WBM). The results are expressed as the mean \pm standard 565 deviation $(\mathrm{n}=3)$, and columns with different letters differ significantly $(p<0.05)$. 566 DMBI = 5,6-dimethylbenzimidazole.

567 Fig. 4. Effect of riboflavin (RF), RF and nicotinamide (NAM), and DMBI 568 supplementation on the volumetric vitamin B12 yield $(\mu \mathrm{g} / \mathrm{mL})($ Fig. 4A) and 569 cellular B12 yield ( $\mu \mathrm{g} / \mathrm{g}$ wet cell mass) (Fig. 4B) by 30 Propionibacterium strains 570 in whey-based medium (WBM). DMBI = 5,6-dimethylbenzimidazole. Error bars 571 represent the standard deviations of three biological replicate fermentations. 572 Strains 256 and 266 were not studied with RF supplementation.

573 The B12 per gram of cells data are not shown for six strains $(261,262,264,265$,

574266 and 289) that were difficult to isolate as cell pellets.

575 Fig. 5. Correlation between riboflavin (RF) consumption and vitamin B12 576 production by 30 strains of Propionibacteria in whey-based medium (WBM). 
A $O D_{600 \mathrm{~mm}}$

B $\mathrm{pH}$

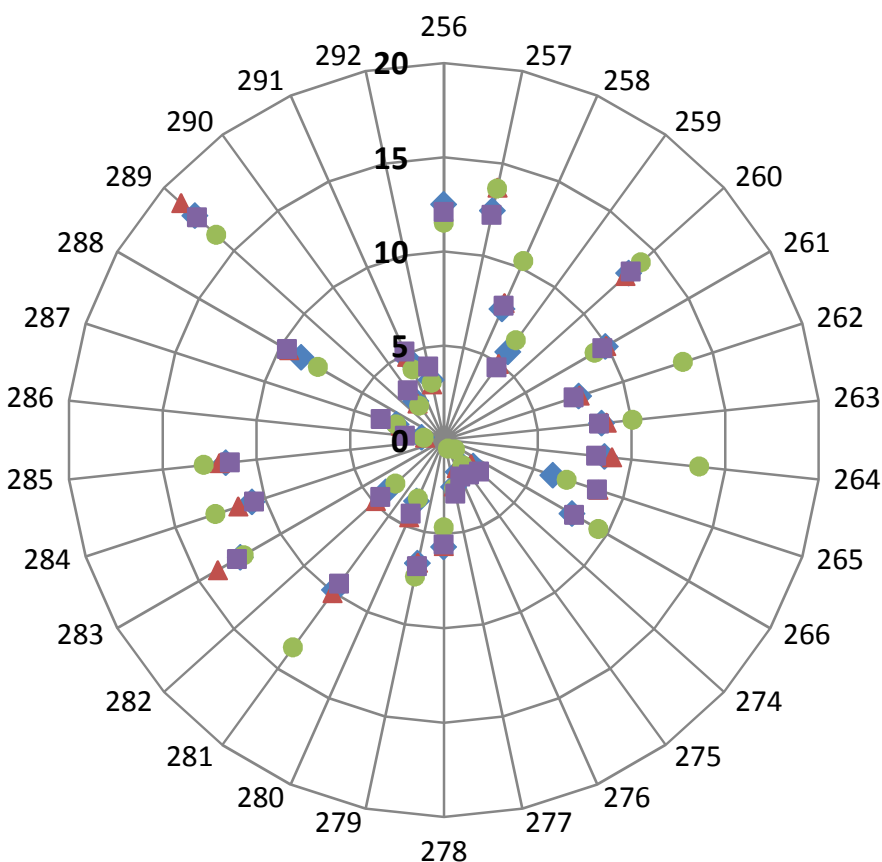

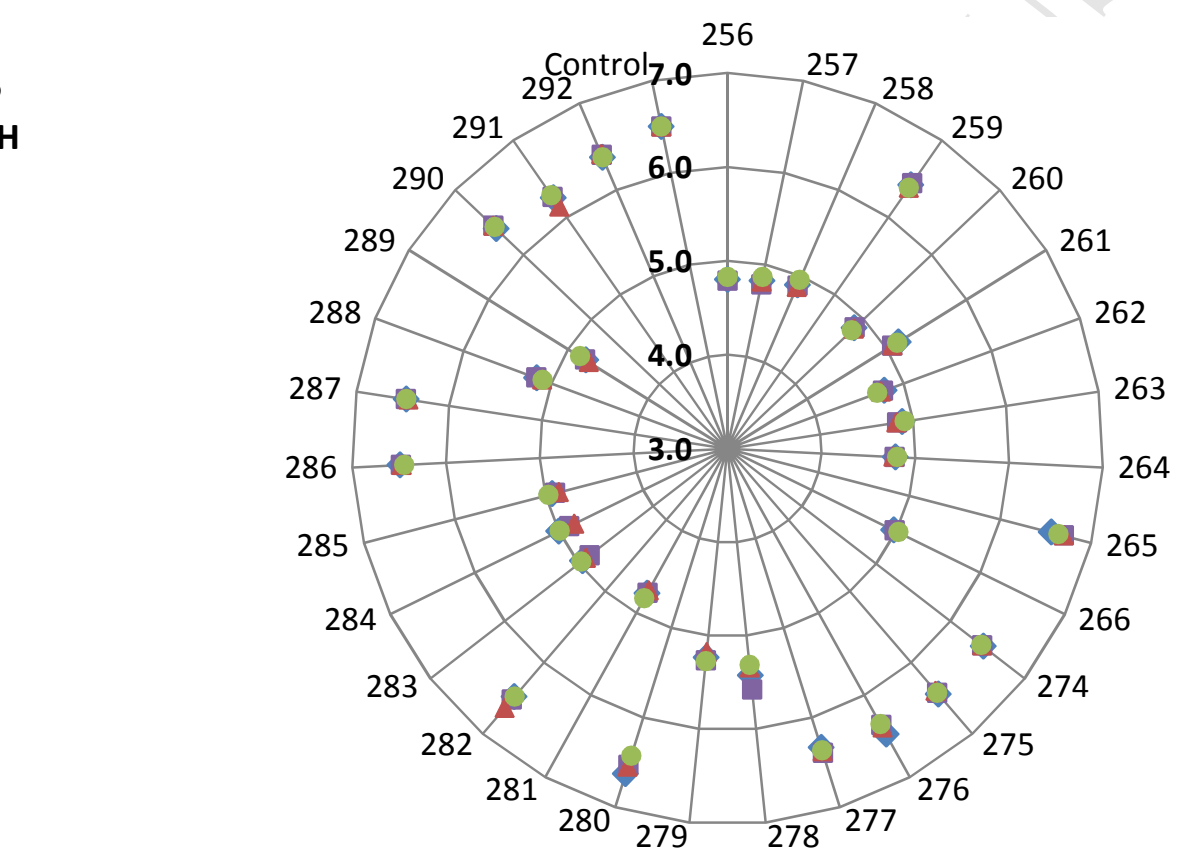

$\checkmark$ WBM

$\triangle$ WBM RF

- WBM RF+NAM

- WBM DMBI 


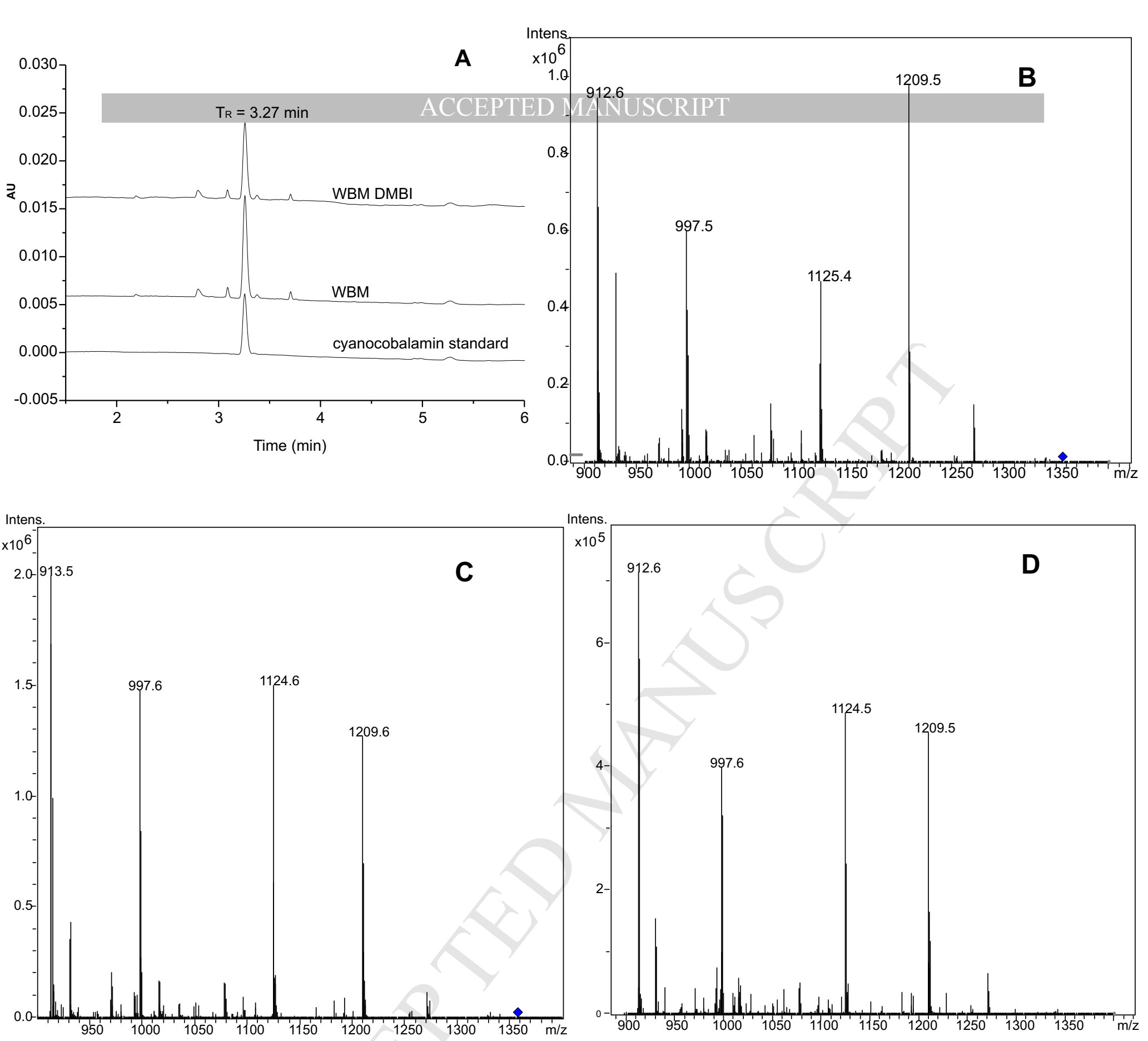




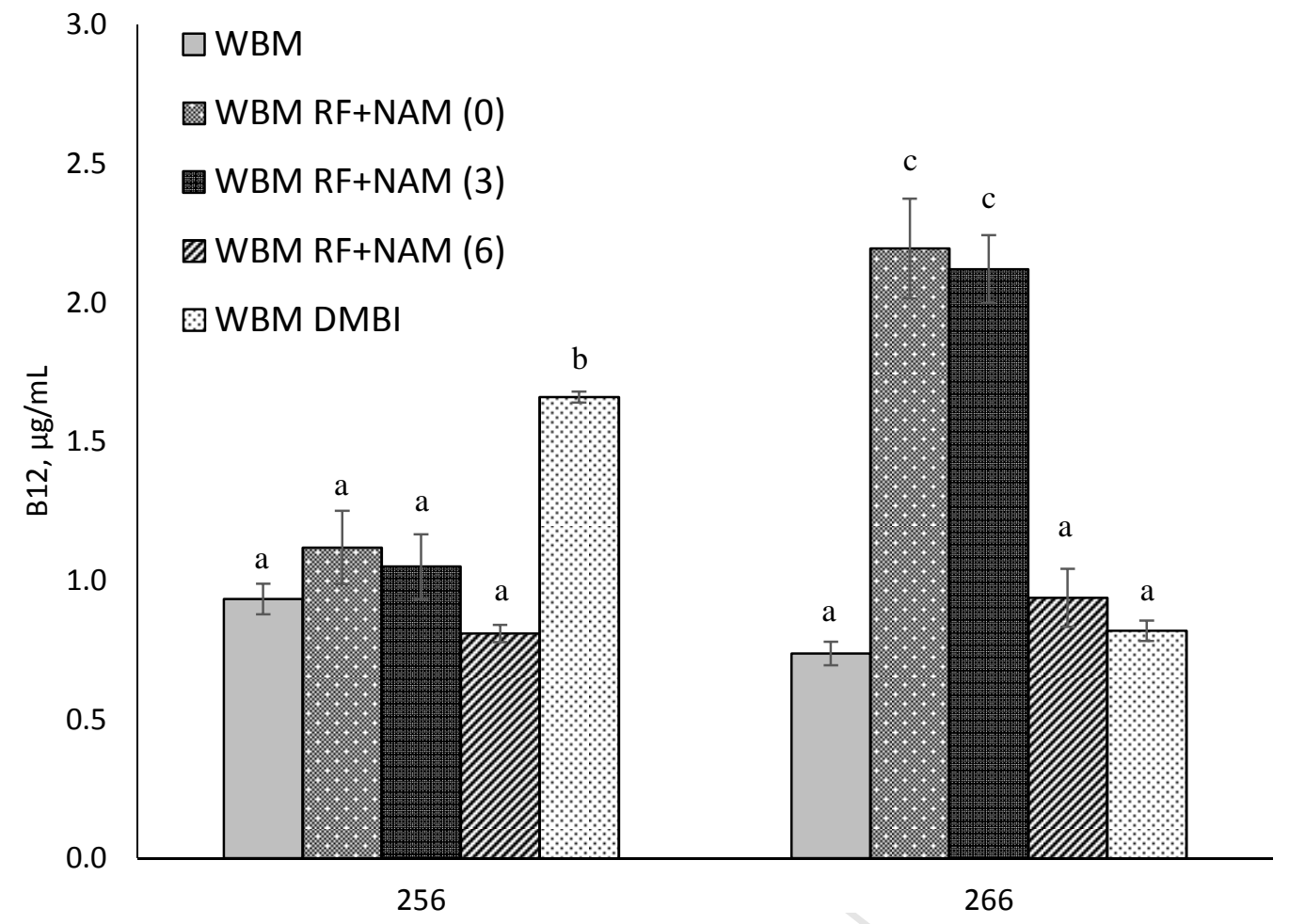



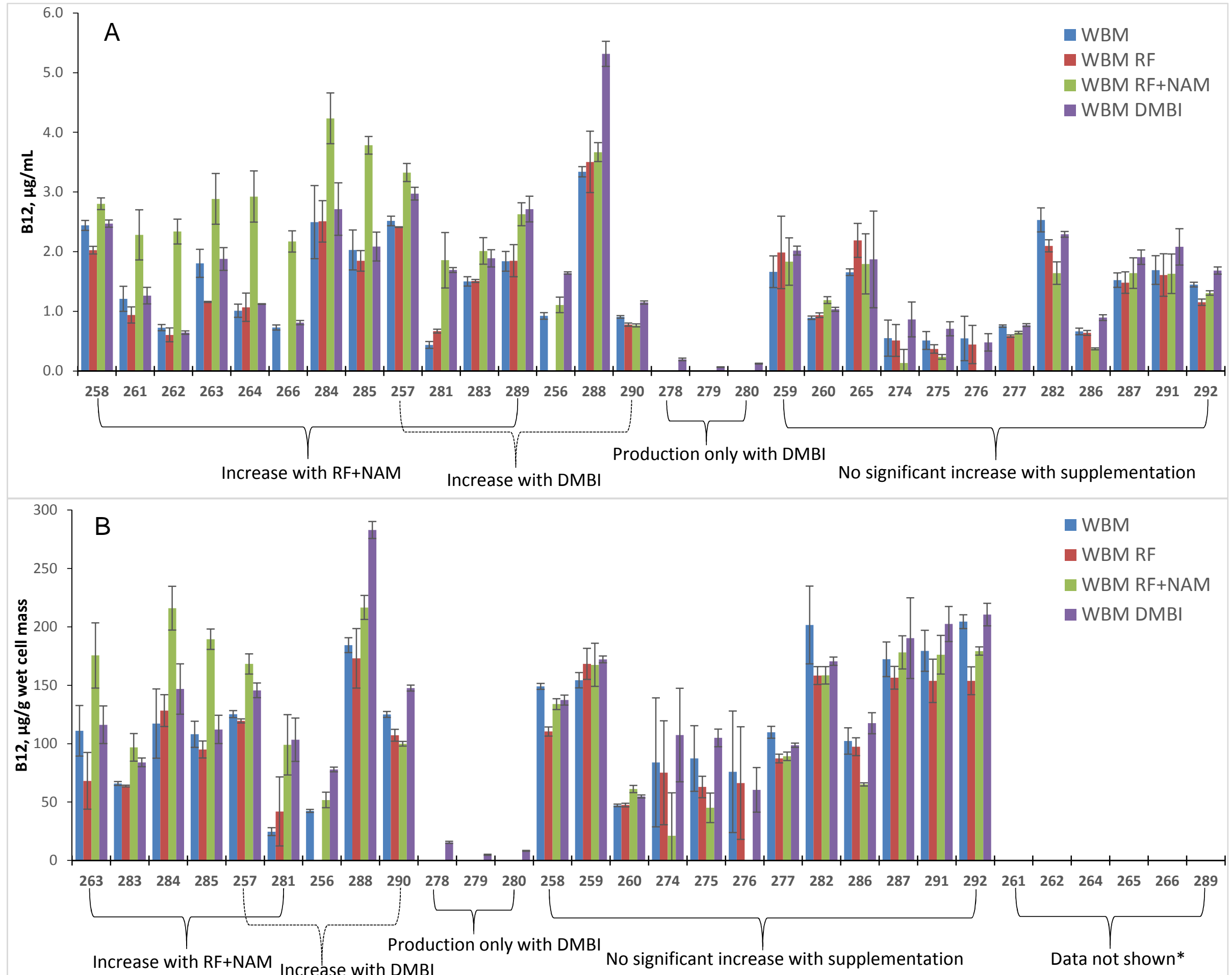


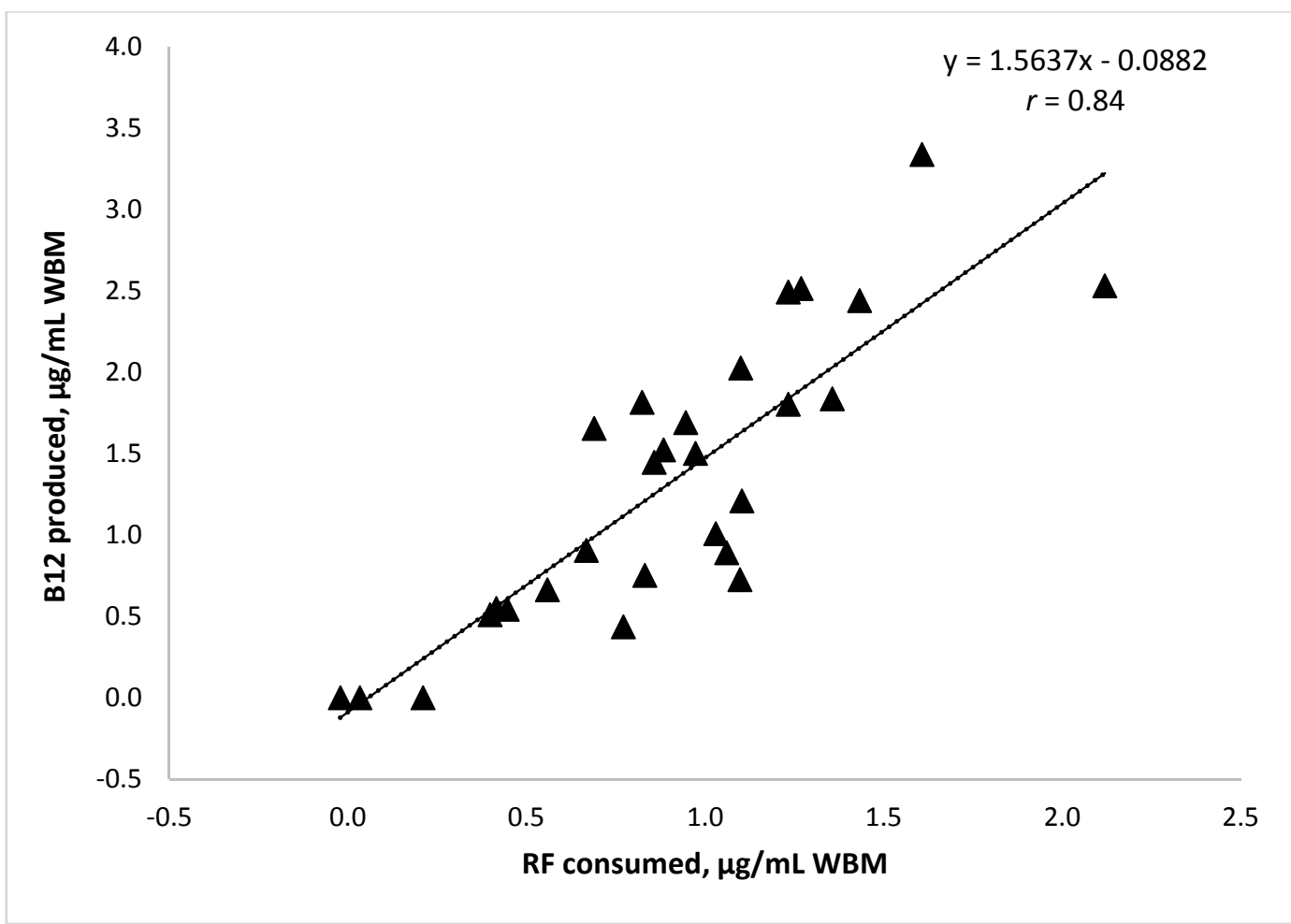


- UHPLC-UV/Vis-MS confirmed active vitamin B12 production by Propionibacterium freudenreichii strains.

- B12 yield was strain dependent.

- Supplementation with riboflavin and nicotinamide increased B12 yield up to 4-fold.

- Significant positive correlation was observed between riboflavin consumption and $\mathrm{B} 12$ production.

- Increasing in situ B12 production is possible with added natural precursors in food matrices. 